\title{
Unlimited expansion of intestinal stem cells from a wide range of ages
}

\author{
Marcin Duleba $^{1}$, Rajasekaran Mahalingam ${ }^{1}$, Audrey-Ann Liew ${ }^{1}$, Yutao Qi $^{1}{ }^{1}$, Rahul Neupane ${ }^{1}$, Matthew Vincent ${ }^{2}$, Suneal Agarwal ${ }^{3}$, Francisco \\ A Sylvester ${ }^{4}$, Jeffrey S Hyams ${ }^{5}$, Khek Yu Ho ${ }^{6}$, Jason K Hou ${ }^{3}$, Frank McKeon ${ }^{1}$ and Wa Xian ${ }^{7,8 *}$ \\ ${ }^{1}$ Department of Biology and Biochemistry, University of Houston, Houston, Texas 77204, USA \\ ${ }^{2}$ Tract Pharmaceuticals, Inc., Marlborough, Massachusetts 01752, USA \\ ${ }^{3}$ Division of Gastroenterology, Baylor College of Medicine, Houston, Texas, USA \\ ${ }^{4}$ Department of Pediatrics, Division of Gastroenterology, The University of North Carolina at Chapel Hill, Chapel Hill, North Carolina 27599, USA \\ ${ }^{5}$ Division of Digestive Diseases, Hepatology, and Nutrition, Connecticut Children's, Medical Center, Hartford, Connecticut 06106, USA \\ ${ }^{6}$ Departments of Medicine and Pathology, National University of Singapore, Singapore \\ ${ }^{7}$ Institute of Molecular Medicine, McGovern Medical School of The University of Texas, Health Science Center, Houston, Texas 77030, USA \\ ${ }^{8}$ Department of Biochemistry and Molecular Biology, University of Texas McGovern, Medical School, Houston, Texas 77030, USA
}

\begin{abstract}
The recent technical advance in cloning and culturing ground-state intestinal stem cells (ISC) provides us an opportunity of accurate assessment of age-related impact on the function of highly proliferative intestinal stem cells. Our ability of indefinitely and robustly expanding single-stem-cell derived pedigrees in vitro allows us to study intestinal stem cells at the clonal level. Interestingly, comparable number of ISC clones was yielded from 1mm endoscopic biopsy of all donors despite the age. They were passaged in vitro as pedigrees and expanded to 1 billion cells in approximately sixty days without changes in stemness demonstrated by clonogenicity and multipotency. Therefore, our study shows that ISCs from a wide range of ages can be cloned and expanded to unlimited number in vitro with similar efficiency and stability. These patient-derived ISCs harbor intrinsic immortality and are ideal for autologous transplantation, supporting the promise of adult-stem-cell based personalized medicine.
\end{abstract}

\section{Introduction}

Autologous transplantation using wild-type or transgenic epidermal stem cells have been proven to be extremely successful in patients with severe burns, chronic wounds and junctional epidermolysis bullosa [1-3]. Conceivably, adult stem cells derived from other regenerative tissues such as intestine can be used to restore the intestinal epithelial functions following autologous transplantation in patients with severe forms of short bowel syndrome (SBS) [4], or those with congenital disorders [5] or those with inflammatory bowel disease (IBD) [6,7].

However, caution needs to be taken when these patient-derived adult stem cells being used for autologous transplantation. Although there is an intriguing amount of evidence suggesting that the stem cells residing in the intestinal tissues of aged people are still quite capable, it is unclear whether their stem cell behavior is similar to those taken from a younger individual. Thus, whether old stem cells are inherently dysfunctional is a question of considerable relevance to the practical development of stem cell therapies based on autologous transplantation for people at all ages.

Cloning, screening and expanding ISCs had been challenging due to our inability of cloning stem cells from columnar epithelial tissue and maintaining their immaturity during in vitro expansion. Consequently,
ISCs had to be carried forward as regenerative, differentiating "organoids" with very low percentage of clonogenic cells, which limits the kinetics of their propagation as well as their utility for exploring the elemental stem cell [8-10].

Recently, a new technology was developed to support cloning the ground state ISCs in their highly immature, clonogenic state. These cultured ground-state ISCs demonstrated remarkable stability in their genome and epigenetic commitment programs, maintained clonogenicity and unlimited replicative expansion, suggesting their tremendous potential for personalized regenerative medicine $[11,12]$.

In this study, we used the ground state stem cell cloning technology to study intestinal stem cells derived from donors in a wide range of ages. We found the behavior of ISCs removed from the old cellular environments was identical to those taken from a younger individual. They can all be passaged robustly and stably in vitro, suggesting the intrinsic immortality of ISCs is age independent.

${ }^{\star}$ Correspondence to: Wa Xian, Institute of Molecular Medicine, McGovern Medical School of The University of Texas, Health Science Center, Houston, Texas 77030, USA, Tel: 1-860-480-1188; E-mail: wa.xian@uth.tmc.edu

Received: June 13, 2019; Accepted: July 05, 2019; Published: July 15, 2019 


\section{Results}

\section{Ground state ISCs derived from patients with a wide range of ages}

In order to understand whether ground-state intestinal stem cells $\left(\mathrm{ISC}^{\mathrm{GS}}\right)$ can be successfully cloned and cultured from patients at all ages, we chose ten patients between age 10 to 20 , ten patients between age 30 to 50 and ten patients between age 50 to 80 (Supplementary Table 1). The $1 \mathrm{~mm}$ biopsies from the intestinal epithelium of these patients were enzymatic digested and seeded in a system including 3T3J2 feeder and specialized medium [11]. We detected approximately 100 colonies that can be derived from each of all thirty patients. Starting from one ISC ${ }^{\mathrm{GS}}$ colony, a billion ISC ${ }^{\mathrm{GS}}$ cells can be generated from all thirty patients independent of age in approximately sixty to eighty days (Figure $1 \mathrm{~A}$ ). The ISC ${ }^{\mathrm{GS}}$ derived from all ages displayed indistinguishable morphology (Figure 1B). In order to test their multipotent differentiation ability, pedigree lines of ISC ${ }^{\mathrm{GS}}$ of 16,42- and 76-years old patients were differentiated in air-liquid interface (ALI) cultures for 10 days. All the ISC $^{\mathrm{GS}}$ formed a highly uniform, 3D serpentine pattern (Figure 1C). Histological sections of these differentiated ISC ${ }^{\mathrm{GS}}$ revealed a columnar epithelium of villuslike structures (Figure 1D, E) marked by goblet (Mucin 2+), endocrine (chromogranin A+), and Paneth cells (defensin alpha 6+) (Figure 1, Supplementary Table 2), indicating that the progeny of a single ISC ${ }^{\mathrm{GS}}$ from a wide range of patients (10yr-76yr) can give rise to all epithelial lineages typically found in the intestine.

A
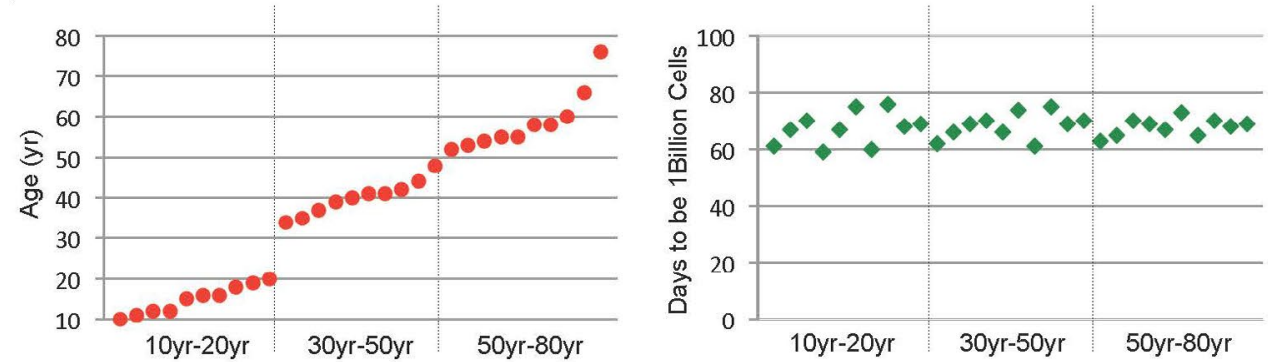

B
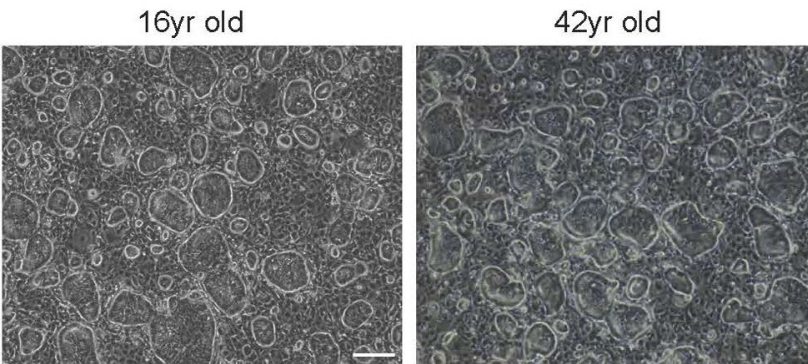

$76 y$ old

C
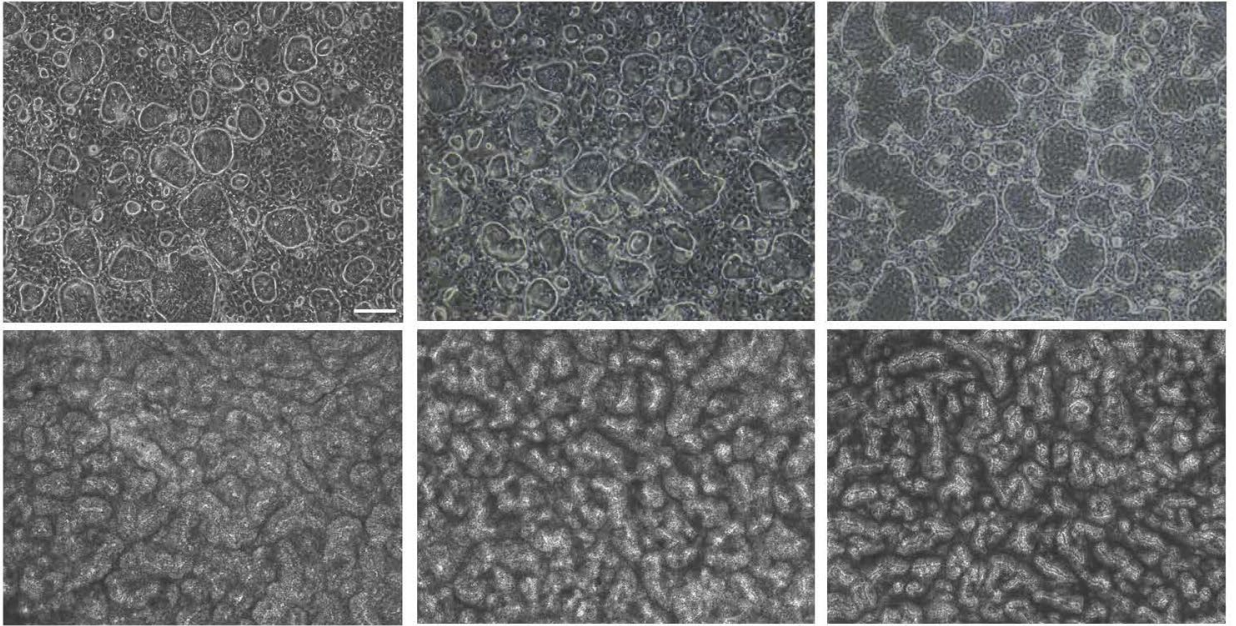

D
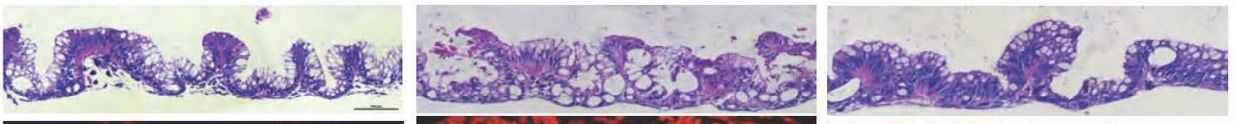

E
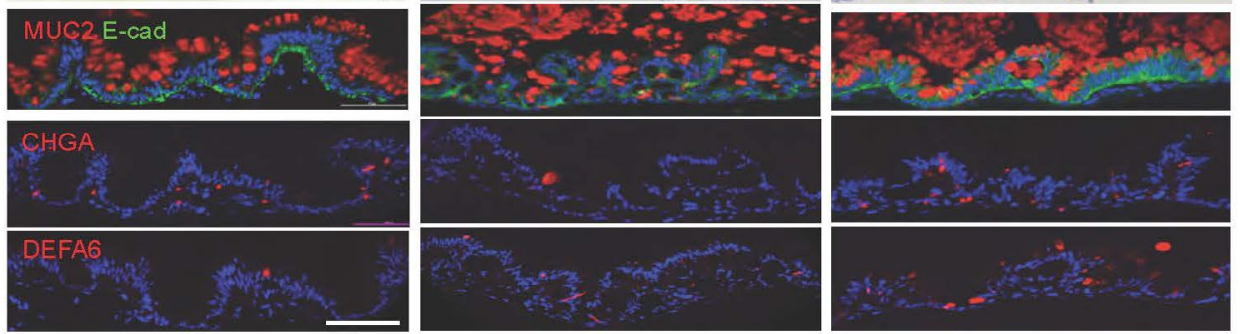

Figure 1. Cloning ISC ${ }^{\mathrm{GS}}$ from patients of wide-range ages

A. left, Histogram depicting ages of the patients included in this study. right, Histogram depicting days needed for single ISC $\mathrm{C}^{\mathrm{GS}}$ from each patient dividing to 1 billion ISCGS. B. Phase contrast images of typical colonies derived from biopsies of $16 \mathrm{yr}, 42 \mathrm{yr}$ and $76 \mathrm{yr}$ old patients. Scale bar, $100 \mu \mathrm{m}$. C. Surface view of ALI cultures. D. Histological sections

through ALI cultures via hematoxylin-eosin staining. E. Immunofluorescence on sections of ALI cultures with indicated antibodies to secretory cell markers Mucin 2, Chromogranin A and Defensin A6. Scale bar, $100 \mu \mathrm{m}$ 


\section{Long-term culturing of ground state ISCs}

We next wanted to study the self-renewal ability of ISCs derived from these thirty patients (10yr-76yr). We first showed that ISC ${ }^{\mathrm{GS}}$ from all patients included in this study are highly clonogenic. Approximately $70 \%$ clonogenicity was observed across the patients (Figure 2A). Due to this high clonogenicity, single cell derived colony can be rapidly expanded to single-cell derived pedigree (Figure 2B). We next examined the functional stability of the ISC ${ }^{G S}$ pedigree in culture. We compared the ISC ${ }^{\mathrm{GS}}$ at early passage (P1) and late passage (P10). Each passage includes culturing in vitro for ten days with approximately 17 cell divisions. Despite passaging number, the stem cells were able to differentiate into intestine-like structures in ALI culture system and expressed differentiation markers such as goblet cell marker (Muc2+) (Figure 2C). Furthermore, these ISC $^{\mathrm{GS}}$ remained high clonogenic ability $(>60 \%)$ despite long-term culturing (Figure $2 \mathrm{D}$ ). This result is consistent with what we observed in human fetal ISC ${ }^{\mathrm{GS}}$ during in vitro expansion [13]. Thus, the stable and robust culturing of ISC ${ }^{\mathrm{GS}}$ is age independent and these cells provide reliable stem cell source for personalized regenerative medicine.

\section{Discussion}

Stem cell based autologous transplantation may improve outcomes of patients with a wide range of disorders of the gastrointestinal tract, characterized by an impaired mucosal barrier function, including IBD, necrotizing enterocolitis, fistulas, NSAID- induced damage, or gastroduodenal bleeding [5,14]. There are critical and unanswered questions relevant to the older patients such as whether the ISC ${ }^{\mathrm{GS}}$ derived from these aged individuals are capable of being expanded faithfully and rapidly to sufficient numbers to functionally regenerate the intestinal epithelium.
A
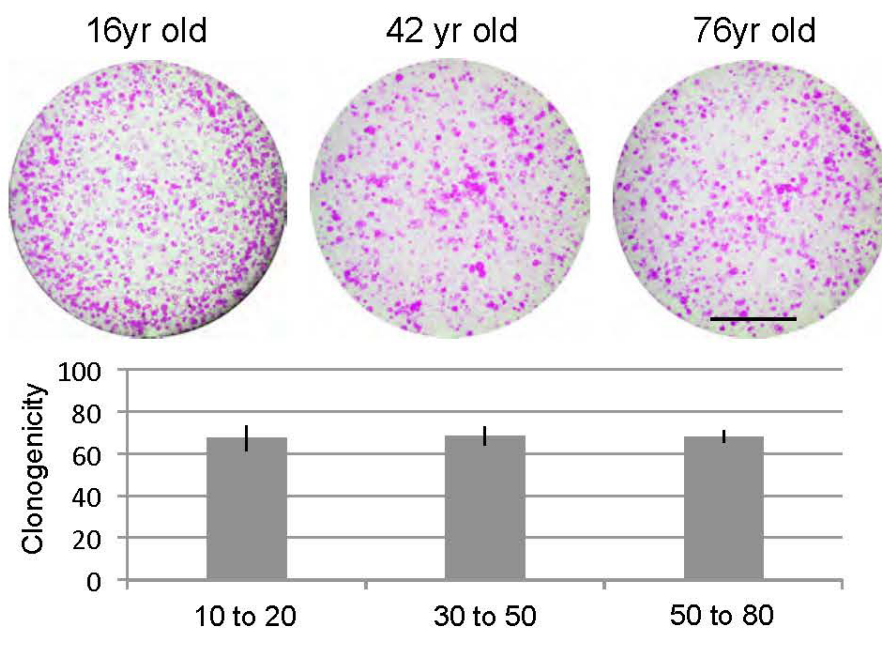

C
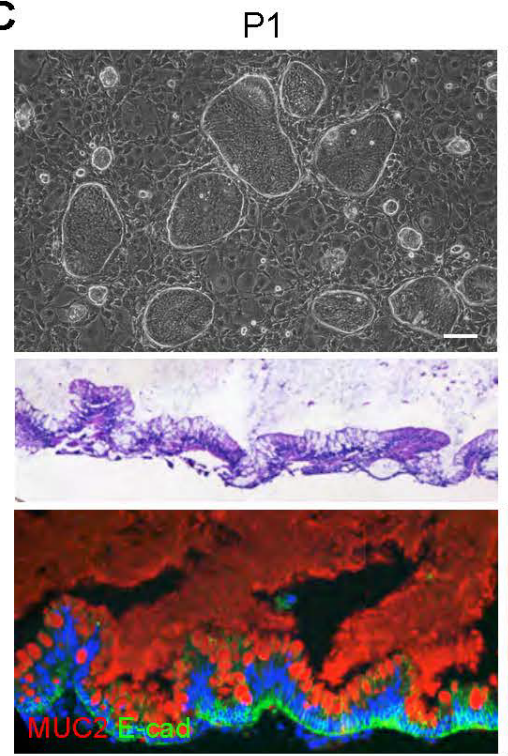

$42 \mathrm{yr}$ old
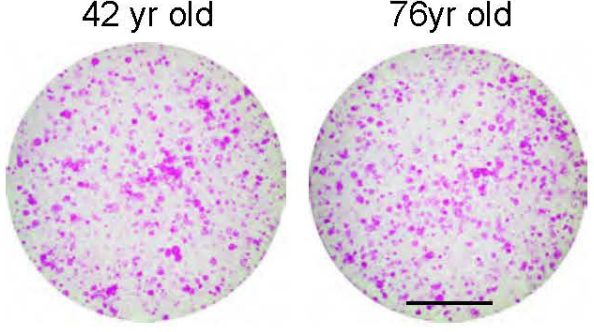

50 to 80
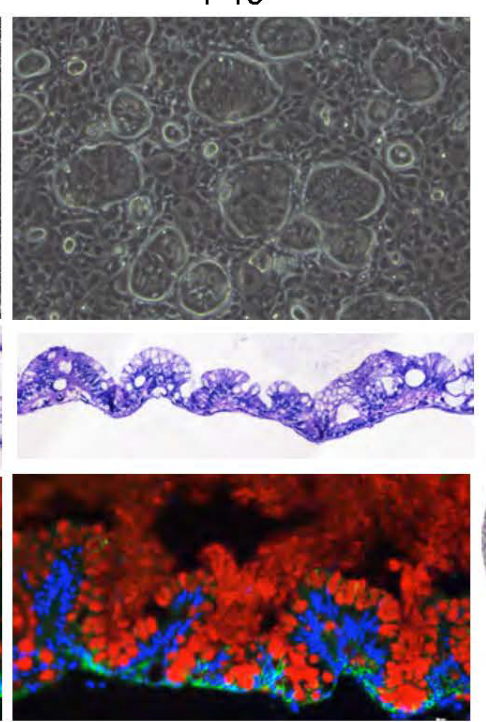

\section{B Single Cell Derived Colony}

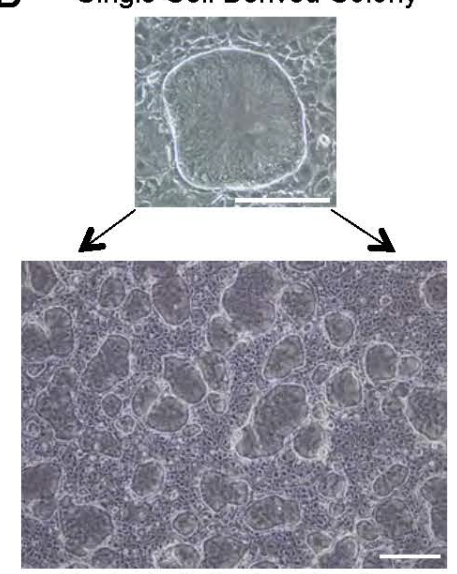

Single Cell Derived Pedigree

D

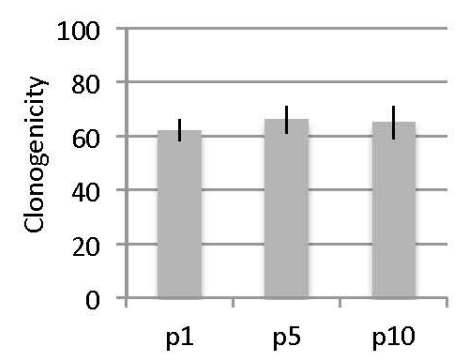

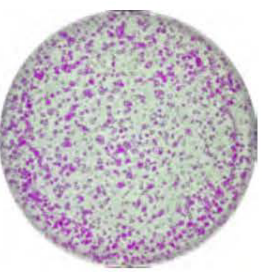

P1

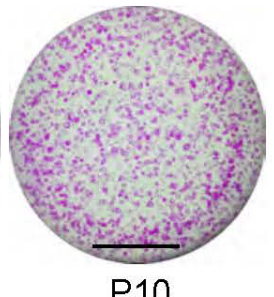

P10

Figure 2. Long-term stability of ISC ${ }^{\mathrm{GS}}$

A. upper, Clonogenicity assay revealing Rhodamine red-stained colonies grown 20days following seeding 1000 ISC ${ }^{\mathrm{GS}}$ cells. Scale bar, 10mm. lower, Quantification of clonogenicity of ground state stem cells from endoscopic biopsy at indicated age range. Error bars, s.d. B. Individual colonies are sampled from the pool and grown as separate lines in isolation. C. upper, Phase contrast images of typical clones at early and late passage of wild-type ISC ${ }^{\text {GS }}$. middle, Histological sections through ALI cultures via hematoxylin-eosin staining. lower, Immunofluorescence on sections of ALI cultures with indicated antibodies to secretory cell markers Mucin 2 . D. Clonogenicity assay of early and late passages of ISC $\mathrm{CSS}^{\mathrm{GS}}$ revealing nearly unchanged number of Rhodamine red- stained colonies grown 10 days following seeding 2,000 passaged ISC ${ }^{\mathrm{GS}}$. 
The current direction in regenerative medicine is to use patient's own stem cells for autologous transplantation. If aged stem cells were inherently dysfunctional, that would greatly limit the ability to use this type of therapies for older people. However, if old stem cells are still maintaining intact stemness, in another words if the intrinsic immortality of ISC $^{G S}$ is age independent, then this approach to regenerative medicine for age-related disease could be very promising. We showed here that ISC ${ }^{G S}$ can be cloned from a wide range of patients aged from 10 to 76 . We did not detect any age- related loss of selfrenewal or differentiation ability. In approximately 60 to 80 days, a single ISC ${ }^{\mathrm{GS}}$ can be expanded to about 1 billion cells among all 30 patients included in this study, suggesting they may serve as ideal stem cell source for autologous transplantation tailored for patients with intestinal disorders.

In 1980s, Howard Green and colleagues demonstrated the first example of cell therapy using cultured adult stem cells. They showed that human epidermis could be grown in the laboratory and transplanted onto burnt patients to reconstitute a functional epidermis $[15,16]$. Since then, this procedure has been shown repeatedly life- saving for patients with severe burns [2]. Furthermore, long-term effectiveness and safety of using genetically modified epidermal stem cells to correct the severe skin blistering disease epidermolysis bullosa has been shown clinically [3]. The successful clinical usage of epidermal stem cells has demonstrated a close correlation with the number of long-lived stem cells used in the procedure that can extensively self-renew in vitro and in vivo $[1,3]$. It remains unclear whether autologous transplantation of cultured intestinal cells can achieve the same success in clinical settings. Although it has been claimed that successful transplantation of organoids including a small fraction of intestinal stem cells can be achieved in murine models of experimental colitis, which shows that these organoids adhere to and become an integrated part of the epithelium [8] it is likely that extremely limited number of stem cells in the organoid structures cannot support the long term intestinal epithelium regeneration in human.

In comparison to less than $1 \%$ presence of intestinal stem cells in the organoids structures, the ground state ISC culture comprises over $60-70 \%$ of ISC ${ }^{G S}$. Based on the previous lessons that we learned through clinical usage of cultured epidermal stem cells, it is conceivable that usage of ISC ${ }^{\mathrm{GS}}$ will significantly improve the efficacy and success of the transplantation. Certainly, another challenging problem is that the influence of the aged or diseased tissue environment must be solved to build effective regenerative therapies for the patient. Nevertheless, with the knowledge that stem cells from elderly patients have similar capabilities to growth and differentiation as those from younger patients and our ability of culturing them in vitro robustly, we foresee a promising future of adult stem cell-based regeneration approaches for patients suffering intestinal disorders.

\section{Methods}

\section{Cell cloning from endoscopic biopsies}

Patients were recruited for this study under informed consent consistent with institutional review board protocols. All biopsies were derived from patient's endoscopy.

$1 \mathrm{~mm}$ endoscopic biopsies were collected into RPMI media (Gibco) with $2 \%$ fetal bovine serum and subsequently digested in $2 \mathrm{mg} / \mathrm{ml}$ collagenase A (Roche) at $37^{\circ} \mathrm{C}$ for 1.5 hour. Cells were washed by centrifugation in RPMI, digested with $0.5 \%$ trypsin (Gibco) $10 \mathrm{~min}$, passed through a 40um Nylon mesh (Falcon), and seeded onto a feeder layer of lethally irradiated 3T3-J2 cells in StemECHO expansion medium and StemECHO enhancer (MultiClonal Therapeutics, West
Hartford, CT). The culture medium was changed every two days. Cells were digested by $0.25 \%$ trypsin and passaged every seven to 10 days.

\section{Stem cell differentiation}

Air-liquid interface (ALI) culture of ISC ${ }^{\mathrm{GS}}$ was performed as described [11,12]. Briefly, cells were cultured on Transwell plates (Corning) in StemECHO expansion medium media. At confluence, the medium on the inserts was removed by careful pipetting and the cultures continued for an additional 10-20 days prior to harvesting. The StemECHO differentiation medium (MCT) was changed every two days. After 2 weeks, the differentiated structures were harvested.

\section{Histology and immunostaining}

Histology and immunofluorescence were performed using standard techniques. For immunofluorescence and immunohistochemistry, $4 \%$ paraformaldehyde fixed, paraffinembedded tissue sections were subjected to antigen retrieval in citrate buffer ( $\mathrm{pH} \mathrm{6.0,} \mathrm{Sigma-Aldrich,} \mathrm{USA)} \mathrm{at} 95^{\circ} \mathrm{C}$ for $20 \mathrm{~min}$, and a blocking procedure was performed with $5 \%$ bovine serum albumin (BSA, Sigma-Aldrich, USA) and $0.01 \%$ Triton X-100 (Sigma-Aldrich, USA) in phosphate-buffered saline (PBS; Gibco, USA) at room temperature for $1 \mathrm{~h}$. Primary antibodies used in this study and staining condition were listed in Supplementary Table 2. All images for section slides were captured by using LSM 510 confocal microscope (Zeiss) with LSM software. Bright field cell culture images were obtained on an Eclipse TS100 microscope (Nikon) with Digital Sight DSFilcamera (Nikon) and NIS- Elements F3.0 software (Nikon).

\section{Acknowledgements}

This work was supported by grants from the Cancer Prevention Research Institute of Texas (CPRIT; RR15014 to WX and RR15088 to FM), the National Institutes of Health (1R01DK115445-01A1 to WX, and U24CA228550 to FM), the US Dept. of Defense (W81XWH-17-1-0123 to WX), the University of Texas Presidential Award (to WX), the American Gastroenterology Association Research and Development Pilot Award in Technology (to WX) and Singapore National Medical Research Council (to KYH and FM). We thank all the members in the Xian-McKeon laboratory for helpful discussions and support.

\section{Conflicts of interest statement}

W.X., F.M., M.D. and M.V. have filed a patent related to the technology used in the present work and Drs. Xian and McKeon have financial interests in MultiClonal Therapeutics Inc.

\section{References}

1. De Luca M, Pellegrini G, Green H (2006) Regeneration of quamous epithelia from stem cells of cultured grafts. Regen Med 1: 45-57. [Crossref]

2. Gallico GG, O'Connor NE, Compton CC, Kehinde O, Green H (1984) Permanent coverage of large burn wounds with autologous cultured human epithelium. New England Journal of Medicine 311: 448-451.

3. Hirsch T, Rothoeft T, Teig N, Bauer JW, Pellegrini G, et al. (2017) Regeneration of the entire human epidermis using transgenic stem cells. Nature 551: 327-332.

4. Buchman AL (2004) The medical and surgical management of short bowel syndrome. MedGenMed 6: 12. [Crossref]

5. Hong SN, Dunn JCY, Stelzner M, Martín MG (2017) Concise Review: The Potentia Use of Intestinal Stem Cells to Treat Patients with Intestinal Failure. Stem Cells Translational Medicine 6: 666-676.

6. Martini E, Krug SM, Siegmund B, Neurath MF, Becker C (2017) Mend your fences: the epithelial barrier and its relationship with mucosal immunity in inflammatory bowel disease. Cell Mol Gastroenterol Hepatol 4: 33-46. [Crossref] 
7. Salim SY, Söderholm JD (2011) Importance of disrupted intestinal barrier in inflammatory bowel diseases. Inflammatory Bowel Diseases 17: 362-381. [Crossref]

8. Fordham RP, Yui S, Hannan NRF, Soendergaard C, Madgwick A, et al. (2013) Transplantation of expanded fetal intestinal progenitors contributes to colon regeneration after injury. Cell Stem Cell 13: 734-744. [Crossref]

9. Matsuura R, Kogo H, Ogaeri T, Miwa T, Kuwahara M, et al. (2006) Crucia transcription factors in endoderm and embryonic gut development are expressed in gutlike structures from mouse ES cells. Stem Cells 24: 624-630. [Crossref]

10. Sato T, Vries RG, Snippert HJ, van de Wetering M, Barker N, et al. (2009) Single Lgr5 stem cells build crypt-villus structures in vitro without a mesenchymal niche. Nature 459: 262-265. [Crossref]

11. Duleba M, Qi Y, Mahalingam R, Liew A (2018) An efficient method for cloning gastrointestinal stem cells from patients via endoscopic biopsies. Gastroenterology 56 : 20-23. [Crossref]
12. Wang X, Yamamoto Y, Wilson LH, Zhang T, Howitt BE, et al. (2015) Cloning and variation of ground state intestinal stem cells. Nature 522: 173-178. [Crossref]

13. Holmberg FE, Seidelin JB, Yin X, Mead BE, Tong Z, et al. (2017) Culturing human intestinal stem cells for regenerative applications in the treatment of inflammatory bowel disease. EMBO Molecular Medicine 9: 558-570.

14. Green H, Kehinde O, Thomas J (1979) Growth of cultured human epidermal cells into multiple epithelia suitable for grafting. Proceedings of the National Academy of Sciences of the United States of America 76: 5665-5668.

15. O'Connor N, Mulliken J, Banks-Schlegel S, Kehinde O, Green H (1981) Grafting of burns with cultured epithelium prepared from autologous epidermal cells. The Lance 317: 75-78. [Crossref]

16. Fukuda M, Mizutani T, Mochizuki W, Matsumoto T, Nozaki K, et al. (2014) Small intestinal stem cell identity is maintained with functional Paneth cells in heterotopically grafted epithelium onto the colon. Genes Dev 28: 1752-1757. [Crossref]

Copyright: (2019 Duleba M. This is an open-access article distributed under the terms of the Creative Commons Attribution License, which permits unrestricted use, distribution, and reproduction in any medium, provided the original author and source are credited. 